\title{
In-situ optical reflectance and synchrotron X-ray topography study of defects in epitaxial dilute GaAsN on GaAs
}

\author{
O. Reentilä $\cdot$ A. Lankinen $\cdot$ M. Mattila $\cdot$ A. Säynätjoki $\cdot$ \\ T. O. Tuomi · H. Lipsanen · L. O'Reilly · P. J. McNally
}

(C) Springer Science+Business Media, LLC 2007

\begin{abstract}
In order to investigate the possibilities of in-situ monitoring of GaAsN bulk layer growth and the crystal quality concerning the formation of misfit dislocations, GaAsN bulk samples with different layer thicknesses were grown using a metal-organic vapor phase epitaxy system equipped with a normal incidence optical reflectance setup. High-resolution X-ray diffractometry and synchrotron radiation X-ray topography were used to characterize the samples after growth. Combining the results from topography images and in-situ reflectance data, the formation of the misfit dislocations can be roughly identified from the reflectance curves and thus observed during growth.
\end{abstract}

\section{Introduction}

There has been an ongoing interest in heteroepitaxial GaAsN/GaAs material structures for applications in optical communication. Metal-organic vapor phase epitaxially (MOVPE) grown GaAsN dilute nitride layers have been examined in the past decade due to the unique effects caused by the addition of nitrogen to the (In)GaAs lattice, see for example Refs. [1] and [2]. In high quality semi-

O. Reentilä $(\square) \cdot$ A. Lankinen · M. Mattila ·

A. Säynätjoki · T. O. Tuomi · H. Lipsanen

Micro and Nanosciences Laboratory, Helsinki University of

Technology, P.O. Box 3500, Micronova, Espoo 02015 TKK,

Finland

e-mail: Outi.Reentila@tkk.fi

L. O'Reilly · P. J. McNally

Research Institute for Networks and Communications

Engineering (RINCE), Dublin City University, Dublin 9, Ireland conductor devices, especially in those that are heterostructure based, a near-perfect crystal lattice is vital. In-situ reflectance monitoring has proven to be a useful tool inbulk layer growth, see for example Refs. [3] and [4]. Here we combine the in-situ reflectance data collected during the growth of dilute $([\mathrm{N}]<1 \%)$ GaAsN bulk layers to ex-situ synchrotron radiation X-ray topography (SR-XRT) [5] results in order to clarify the formation of misfit dislocations (MDs) and the observation of their formation during the growth.

\section{Experimental}

The samples were grown by a low-pressure MOVPE system on $350 \mu \mathrm{m}$-thick, (100)-oriented semi-insulating GaAs substrates having a miscut angle of less than $0.5^{\circ}$. Two of the samples were grown on double side polished (DS) GaAs wafers while other two were grown on single side polished (SS) wafers. Growth temperature was $575{ }^{\circ} \mathrm{C}$ (a thermocouple reading), growth pressure 100 torr and susceptor rotation speed $100 \mathrm{rpm}$ during the fabrication of all the samples. Tertiarybutylarsine (TBAs), trimethylgallium (TMGa) and dimethylhydrazine (DMHy) were used as precursors for arsenic, gallium and nitrogen, respectively. To fabricate samples with the same nitrogen content and with different GaAsN layer thicknesses, all flows to the reactor were kept the same during the growth of all the samples and only the growth times were varied. The growth rate of the GaAsN layers was approximately $0.33 \mathrm{~nm} / \mathrm{s}$. No extra capping layer was grown on top of the GaAsN layers, nor have the samples experienced any thermal annealing or other treatment after growth to affect their crystal quality or characteristics. Table 1 lists the relevant properties of the samples. 
Table 1 The growth times of the $\mathrm{GaAs}_{1-x} \mathrm{~N}_{x}$ epilayers, their measured thicknesses and their nitrogen contents

\begin{tabular}{lllll}
\hline & $\begin{array}{l}\text { GaAsN } \\
\text { growth time }(\mathrm{s})\end{array}$ & $\begin{array}{l}\text { GaAsN } \\
\text { thickness }(\mathrm{nm})\end{array}$ & $\begin{array}{l}\text { Nitrogen } \\
\text { content }(\%)\end{array}$ & $\begin{array}{l}\text { Substrate } \\
\text { type }\end{array}$ \\
\hline Sample A & 638 & 210 & 0.86 & DS \\
Sample B & 1,376 & 480 & 0.85 & DS \\
Sample C & 1,420 & 470 & 0.83 & SS \\
Sample D & 1,640 & 560 & 0.86 & SS \\
\hline
\end{tabular}

In the substrate type column, DS means double side polished and SS single side polished substrates

The in-situ monitoring of growth was realized by a normal incidence reflection setup with a halogen lamp as a light source. The reflected signal was detected at $635 \mathrm{~nm}$. Part of the light at $635 \mathrm{~nm}$ is reflected from the GaAs(N) surface and some of it is absorbed into the material, forcing us to utilize the complex refractive index $n_{c}=n+i \kappa$ in order to model the in-situ reflectance data.

The thicknesses and nitrogen contents of the GaAsN layers were determined by measuring $\omega-2 \theta$ diffraction curves using high-resolution X-ray diffraction (HR-XRD) and fitting simulations to the measured data. The simulation software was based on solving the Takagi-Taupin equations for dynamical diffraction occurring in a multilayer structure [6].

SR-XRT measurements were executed in order to investigate dislocations in the GaAsN layers. The SR-XRT measurements were carried out at the HASYLAB-DESY (Hamburger Synchrotronstrahlungslabor am Deutschen Elektronen-Synchrotron) in Hamburg at the F1 Topography beam line using a continuous spectrum of wavelengths from one of the bending magnet sources in the DORIS synchrotron ring. The topographs were recorded on 100 $\mathrm{mm} \times 120 \mathrm{~mm}$ Slavich VRP-M high-resolution holographic X-ray films in the back-reflection geometry with a film-to-sample distance of $60 \mathrm{~mm}$. The (001) surfaces of the samples were first aligned perpendicularly to the square-shaped incident beam having a size of $1.4 \mathrm{~mm} \times$ $1.4 \mathrm{~mm}$, after which the samples were tilted $6^{\circ}$ around the horizontal axis, which was perpendicular to the incoming beam, in order to ensure that the 004 diffractions were recorded on the films. The epitaxial GaAsN layers were facing the film side in all of the exposures.

Additionally, the sample surfaces were micrographed with atomic force microscopy (AFM) in order to acquire data on the surface quality of the samples. The image size in AFM micrographs was $10 \mu \mathrm{m} \times 10 \mu \mathrm{m}$.

\section{Results and discussion}

Figure 1 shows the XRD measurements from the samples and simulations fitted to each of them. According to the
XRD measurements and the corresponding simulations, the nitrogen contents of the layers vary from 0.83 to $0.86 \%$ and the layers are $210-560 \mathrm{~nm}$ thick. The effect of relaxation of the layers on the simulated nitrogen content was assumed to be negligible and therefore all the simulations were made without relaxation.

Figure 2 shows the in-situ reflectance data measured during MOVPE growth of the GaAsN layers and two simulation curves calculated by a well-known matrix method. Vertical offset is added for clarity. During the simulation process the growth rates of the layers were fixed to the true growth rates obtained by the XRD measurements. In addition, $n_{c}$ of GaAs was fixed to $4.00-i 0.30$ at the growth temperature $\left(575^{\circ} \mathrm{C}\right)$ [7]. By fitting, the complex refractive index of GaAsN was found to be approximately $n_{c, \text { GaAsN }}=3.99-i 0.31$.

The noisy appearance of the measured curves is caused by the small complex refractive index difference between GaAsN and GaAs, which means that the changes in the reflectance curves due to the growth of the GaAsN layer are small. In an optimal growth situation the reflectance signal should appear as a damped but averagely constant Fabry-Perot oscillation signal (see simulated curves in Fig. 2). However, during the growth of the three thickest GaAsN samples, B, C, and D, the reflectance curves experience a sudden change in their form, which occurs about 2,100 s after the beginning of the growth process. It corresponds to a layer thickness of approximately $270 \mathrm{~nm}$ assuming a growth rate of $0.33 \mathrm{~nm} / \mathrm{s}$. Sample A being the thinnest and having a thickness of only $210 \mathrm{~nm}$ does not experience such a change in the reflectance oscillation.

An explanation for the sudden change in the reflectivity, notwithstanding the differences in the exact form of the deviation from the simulated reflectivity curves between the samples, might lie in a change in the GaAsN/GaAs interface. The deviation from the simulated reflectivity values is likely to be caused by changes in the sample structure after 2,100 s of growth. This theory is also supported by the observation that the oscillations in curves $\mathrm{C}$ and $\mathrm{D}$ disappear at $2,100 \mathrm{~s}$ indicating that the interface between the GaAs substrate and the GaAsN layer is 


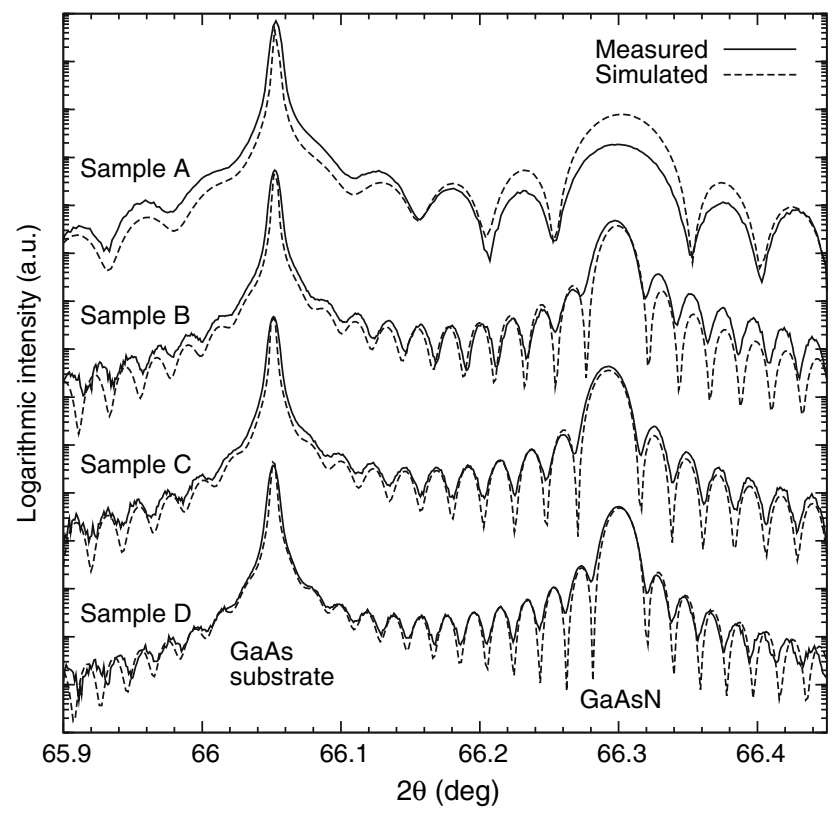

Fig. 1 XRD measurement curves (measured in $\omega-2 \theta$ configuration) and the corresponding simulations fitted to them. The vertical offset between the curves is added for clarity

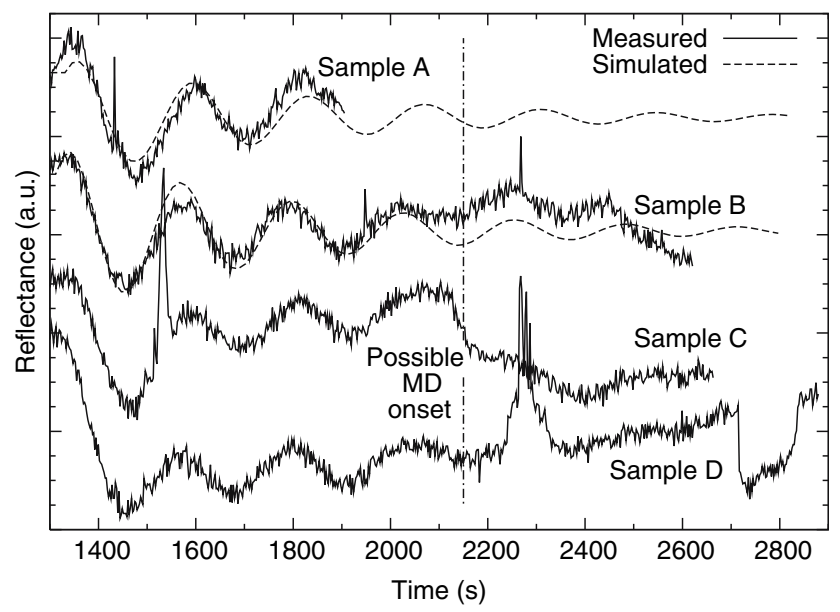

Fig. 2 In-situ reflectance curves measured during the growth of the samples. Simulated curves for samples A and B are plotted for comparison with measured values. The GaAsN epilayers start to grow at about $1,300 \mathrm{~s}$ after the start of the growth run

changed and thus the incoming light is only reflected from the GaAsN surface.

It has been observed that in the InP/InGaP system the formation of the MD networks does indeed happen during growth and not during the cool down after growth [8]. We believe that the same happens here, because it is expected that the thermal expansion coefficient for these dilute GaAsN layers is close to that of GaAs. We suspect that the formation of the MD network during the growth causes the changes in the GaAsN/GaAs interface and thus the changes in the reflectance curves at 2,100 s. This possible beginning of the development of the MD network formation is denoted in Fig. 2.

Figure 3 shows 004 large-area back-reflection SR-XRT images of samples A, B, C and D. Fig. 3a proves the absence of MDs in the $210 \mathrm{~nm}$-thick GaAsN sample A, whereas all thicker samples contain MDs in the interfaces between GaAs and GaAsN. Of particular interest is the sample B having a $480 \mathrm{~nm}$-thick epilayer, because the number of the MD lines intersecting the [110] direction is only $100 \mathrm{~cm}^{-1}$ and the number of the MDs intersecting the [110] direction is negligible. In the other thick samples the number of the MD lines is about $600 \mathrm{~cm}^{-1}$ in both directions. In all of the samples, the MD lines were very long, most of them over $1 \mathrm{~mm}$. The dislocation densities for samples $\mathrm{C}$ and D were confirmed by the AFM micrographs, which showed the MDs as a cross-hatched pattern of ridges having a height of less than $1 \mathrm{~nm}$ on the sample surfaces. Sample B had so few MDs that observing them with AFM was not feasible. The GaAsN surfaces in all of the AFM micrographs appeared atomically flat with no other defects than the misfit ridges visible. The small number of MDs in sample B may be the reason why the in situ reflectance curve in Fig. 2 deviates only slightly from the theoretically calculated one, despite the existence of MDs in the sample. Additionally, topographs in Fig. 3 show that all the samples contain threading dislocations, the endpoints of which are visible in the topographs.

Figures $3 \mathrm{c}$ and $\mathrm{d}$ also show defects other than MDs. Those other defected areas appear as dark stripes between the MD line images that are seen as white stripes in the topographs. This appearance of additional defects to the GaAsN/GaAs interface might be the explanation for the disappearance of the oscillations in the in-situ reflectance curves of samples $\mathrm{C}$ and $\mathrm{D}$. It should be noted that for sample $\mathrm{B}$, these additional defected areas are not seen and that the oscillation at the reflectance curve of sample B continues, even though the reflectance curve slightly deviates from the theoretical reflectance curve.

MDs in the GaAsN/GaAs interface are caused by the slight difference between the lattice constants of $\mathrm{GaAs}_{1-x} \mathrm{~N}_{x}$ and GaAs. The GaAs $\mathrm{s}_{1-x} \mathrm{~N}_{x}$ lattice constant can be interpolated linearly from cubic $\mathrm{GaN}$ and GaAs lattice constants $\quad\left(a_{\mathrm{GaAs}}=5.65359 \AA\right.$ and $a_{\mathrm{GaN}}=4.5315 \AA$ at $300 \mathrm{~K}$ [9]) using Vegard's law [10]. The tensile lattice misfit $f$ for $\mathrm{GaAs}_{1-x} \mathrm{~N}_{x}$ on GaAs is

$f=\frac{\left|a_{\mathrm{GaAs}}-a_{\mathrm{GaAs}_{1-\mathrm{x}} \mathrm{N}_{\mathrm{x}}}\right|}{a_{\mathrm{GaAs}}} \approx 0.2476 x$,

which for the dilute GaAsN layers in this work $([\mathrm{N}] \approx$ $0.85 \%$ ) corresponds to $f \approx 2.1 \cdot 10^{-3}$. Thus, the small lattice 

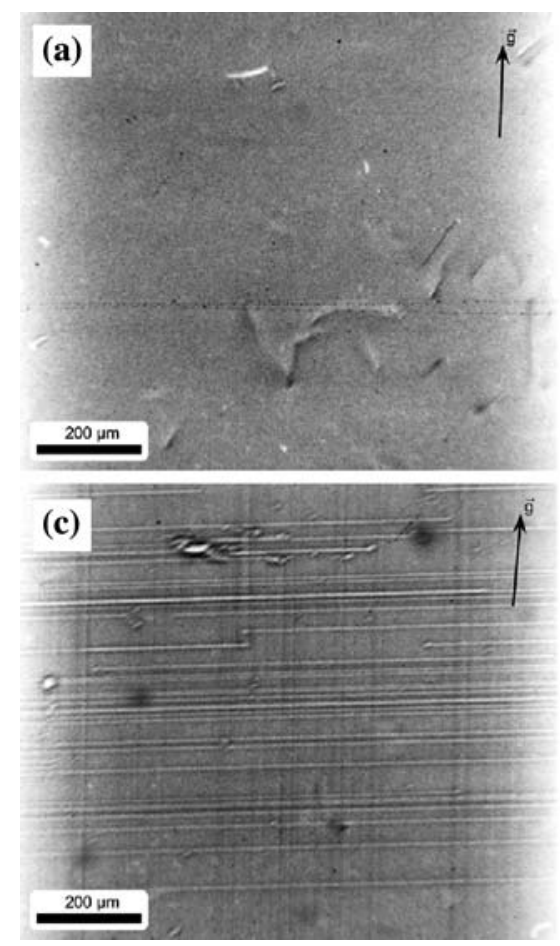

Fig. 3004 large-area back-reflection SR-XRT images of samples (a) $\mathrm{A}$, (b) B, (c) $\mathrm{C}$ and (d) D with related diffraction vector projections $\vec{g}$. Misfit disloca tions are clearly visible in (b) (c) and (d); whereas

misfit should enable the deposition of relatively thick dilute $\mathrm{GaAs}_{0.9915} \mathrm{~N}_{0.0085}$ epilayers on GaAs.

A model for critical thickness by Matthews and Blakeslee utilizing mechanical equilibrium theory gives a critical thickness of $[11,12]$

$d_{c} \approx \frac{b}{2 \pi f}\left[\frac{1-v \cos ^{2} \theta}{(1+v) \cos \lambda}\right]\left[\ln \left(\frac{d_{c}}{b}\right)+1\right]$,

where $v$ is the substrate Poisson ratio (for GaAs, $v \approx 0.320$ [13]), $b=a / \sqrt{2}$ is the slip distance for the MDs, $\theta$ is the angle between the MD line direction $\vec{u}$ and the MD Burgers vector $\vec{b}$ and $\lambda$ is the angle between $\vec{b}$ and $\vec{u} \times \vec{n}$, where $\vec{n}$ is the sample surface normal. Assuming misfit relaxation by $60^{\circ} \frac{1}{2}\langle 101\rangle\{111\}$ MDs (i.e. $\cos \theta=\cos \lambda=1 / 2$ ) the model gives a critical thickness of $d_{c} \approx 320 \mathrm{~nm}$ for the samples A, $\mathrm{B}, \mathrm{C}$ and D. However, assuming that the MDs are of $\frac{1}{2}\langle 110\rangle\{111\}$ pure edge type (i.e. $\cos \theta=0$ and $\cos \lambda=1$ ), the model yields a significantly smaller critical thickness $d_{c}$ $\approx 160 \mathrm{~nm}$ for the same samples.

The observed critical thickness based on SR-XRT of the samples was between 210 and $500 \mathrm{~nm}$, because there were no misfit dislocations in sample A, but MD networks were present in samples B, C, and D. Because sample B had so few misfit dislocations (in some of the topographed areas the distance between adjacent MDs was more than 0.5
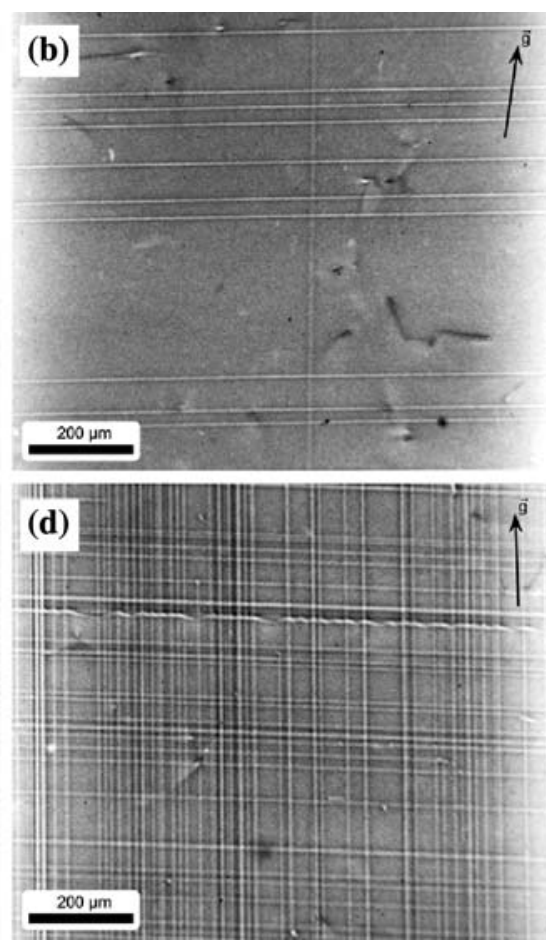

topograph a) shows the absence of misfit dislocations in the $210 \mathrm{~nm}$ thick GaAsN sample A. The black horizontal dotted line in (a) is a scratch on the film.

$\mathrm{mm}$ ), it is believed to exceed the critical thickness $c_{d}$ by a very small amount. Samples B and C have almost the same thickness, but according to the topographs in Fig. 3 sample $\mathrm{C}$ contains a far greater number of MDs. Even though it is not known why sample B had fewer MDs, the topography is consistent with the in-situ data of Fig. 2, where the reflectance curve of sample B is seen to continue oscillating almost to the end of the growth run. This phenomenon is believed to be connected to the absence of defected areas and very low MD density of sample B seen in the topographs. Thus, it appears that samples $\mathrm{C}$ and $\mathrm{D}$ developed a denser MD network earlier than sample B.

Most of the MDs in thin film III-V heteroepitaxy are either pure edge $\frac{1}{2}\langle 110\rangle\{111\}$ dislocations or $60^{\circ} \frac{1}{2}\langle 101\rangle\{111\}$ mixed dislocations $[14,15]$. All of the back-reflection topographs on the film recorded from samples B and D were examined with a microscope for the Burgers vector analysis. Using the information about the diffraction vector $\vec{g}$ of the observed reflections together with the diffraction disappearance criterion

$\vec{g} \cdot \vec{b}=0$

for Burgers vector $\vec{b}$, it was determined that any pure edge dislocations having their Burgers vectors $\vec{b}$ of either type $\pm[110]$ or $\pm[1 \overline{1} 0]$ should disappear in topographs having 
diffraction vectors $\vec{g}= \pm[1 \overline{1} z]$ or $\vec{g}= \pm[11 z]$ for any $z$, respectively. Such diffractions for $z=4,5,7$, and 9 were present in the film recorded from sample B, but no MD image disappearance was detected, even though the topographs were of good image quality. Also, pure edge MDs images in 004 topographs of Fig. 3 should similarly disappear, which is clearly not the case. Therefore, the observed MDs could not be of the pure edge type. Thus, it is most likely that the MDs in samples B, C and D are typical $60^{\circ} \frac{1}{2}\langle 101\rangle\{111\}$ mixed MDs, which are indeed easily generated in heteroepitaxial thin films [14, 16]. Specifically, in Ref. [14] it was observed that heteroepitaxial Si/ GeSi structures having lattice misfit $0.1 \% \leq f \leq 2.0 \%$ contained predominantly $60^{\circ} \frac{1}{2}\langle 101\rangle\{111\}$ mixed MDs near the critical thickness $d_{c}$, which is a very similar result but for group IV materials. Also, the calculated theoretical critical thickness $c_{d} \approx 320 \mathrm{~nm}$ for $60^{\circ} \frac{1}{2}\langle 101\rangle\{111\}$ mixed MDs appears to be in a reasonable agreement with the 480nm-thick sample B considering the observed very few MDs in the sample. However, the reason why the $60^{\circ}$ MDs are preferential in the dilute $\mathrm{GaAsN} / \mathrm{GaAs}$ material system could not be explained.

The observation that there were no pure edge MDs in the samples might first seem rather strange. After all, the $60^{\circ}$ mixed MDs are known to have a smaller stress-relaxing effect than the pure edge MDs, because the screw component of the $60^{\circ}$ dislocations does not relax interfacial stress. However, the thin and dilute GaAsN layers obviously have quite a low tensile stress, so it is possible that even a small number of $60^{\circ}$ mixed MDs is enough to relax the stress. Thus, we propose that near the critical thickness the MD formation in GaAsN/GaAs is dominated by the $60^{\circ}$ mixed MDs. Considering the SR-XRT results in Fig. 3, it is obvious that the growth runs succeeded better on the double side polished substrates, as there were fewer MDs on sample B than on sample $\mathrm{C}$ even though they have practically the same thickness. So, it is possible that even the best sample B might not have reached the attainable theoretical critical thickness. Nevertheless, the small theoretically predicted critical thickness for pure edge MD formation and the observed absence of pure edge MDs in the samples are facts supporting the hypothesis of the MD formation beginning exclusively with the $60^{\circ}$ mixed MDs in the dilute GaAsN on GaAs interface.

It should be noted that the theoretical value of the critical thickness $c_{d}$ strongly depends on the accuracy of the determination of the $\mathrm{GaAs}_{1-x} \mathrm{~N}_{x}$ lattice constant, which makes the analysis difficult. According to Ref. 17 a method based on linear interpolation of the unit cell volumes of cubic GaAs and GaN gives a better accuracy for approximating the $\mathrm{GaAs}_{1-x} \mathrm{~N}_{x}$ lattice constant than using Vegard's law for small nitrogen contents $x$. Inserting the lattice constant acquired from the linear interpolation of unit cell volume approximation with nitrogen content $x=0.85 \%$ into Eqs. (1) and (2) yields a critical thickness $c_{d}=530 \mathrm{~nm}$ for the $60^{\circ}$ mixed MDs, which is larger than both the experimentally observed critical thickness and the critical thickness acquired using Vegard's law. Furthermore, the authors of a more recent study in Ref. [10] confirmed the validity of Vegard's law for GaAsN/GaAs with small nitrogen content.

\section{Conclusions}

Dilute GaAsN layers with different thicknesses were grown by MOVPE and the growth was monitored by in situ reflectometry. By ex-situ SR-XRT measurements the presence of misfit dislocations was observed in three of the four samples. Changes in the measured in-situ reflectance curves compared to the theoretical curves appear after a certain growth time and the changes are observed to correlate with the MD formation at the GaAsN/GaAs interface.

$60^{\circ}$ mixed MDs were detected in all but one of the samples. The observed critical thickness of the GaAsN layers was slightly larger than the expected theoretical value. It was observed that the MD network generation in GaAsN/GaAs material system starts with the formation of $60^{\circ}$ mixed MDs under a relatively low tensile strain $(f \approx$ $0.21 \%)$.

Acknowledgements This work was supported by the European Community - Research Infrastructure Action under the FP6 "Structuring the European Research Area'" Programme (through the Integrated Infrastructure Initiative "Integrating Activity on Synchrotron and Free Electron Laser Science',.)

\section{References}

1. M. Kondow, K. Uomi, A. Niwa, T. Kitatani, S. Watahiki, Y. Yazawa, Jpn. J. Appl. Phys. 35, 1273-1275 (1996)

2. J. Toivonen, T. Tuomi, J. Riikonen, L. Knuuttila, T. Hakkarainen, M. Sopanen, H. Lipsanen, P.J. McNally, W. Chen, D. Lowney, J. Mat. Sci. Mat. El. 14, 267-270 (2003)

3. H. Hardtdegen, N. Kaluza, R. Schmidt, R. Steins, E.V. Yakovlev, R.A. Talalaev, Yu.N. Makarov, J.-T. Zettler, phys. stat. sol. a 201, 312-319 (2004)

4. A. Rebey, M.M. Habchi, A. Bchetnia, B. El Jani, J. Cryst. Growth 261, 450-457 (2004)

5. T. Tuomi, K. Naukkarinen, P. Rabe, phys. stat. sol. a 25, 93-106 (1974)

6. M.A.G. Halliwell, M.H. Lyons, M.J. Hill, J. Cryst. Growth 68 , 523-531 (1984)

7. O. Reentilä, M. Mattila, M. Sopanen, H. Lipsanen, J. Appl. Phys. 101, 033533 (2007)

8. S. Hasenöhrl, R. Kúdela, J. Novák, T.O. Tuomi, L. Knuuttila, Materials Science and Engineering B 88, 134-138 (2002)

9. O. Madelung (eds.), Data in Science and Technology, Semiconductors Group IV Elements and III-V Compounds (Springer, Berlin 1991) 
10. K. Volz, O. Rubel, T. Torunski, S.D. Baranovskii, W. Stolz, Appl. Phys. Lett. 88, 081910 (2006)

11. J.W. Matthews, A.E. Blakeslee, J. Cryst. Growth 27, 118-125 (1974)

12. D.J. Paul, Semicond. Sci. Technol. 19, R75-R108 (2004)

13. B.F. Usher, D. Zhou, S.C. Goh, T. Warminski and X.P. Huang, Optoelectronic and Microelectronic Materials and Devices, 1998. Proceedings 290-293 (1999)
14. D.J. Eaglesham, D.M. Maher, E.P. Kvam, J.C. Bean, C.J. Humphreys, Phys. Rev. Lett. 62, 187-190 (1989)

15. Y.H. Lo, Appl. Phys. Lett. 59, 2311-2313 (1991)

16. J. Narayan, S. Oktyabrsky, J. Appl. Phys. 92, 7122-7127 (2002)

17. M. Reason, X. Weng, W. Ye, D. Dettling, S. Hanson, G. Obeidi, R.S. Goldman, J. Appl. Phys. 97, 103523(2005) 